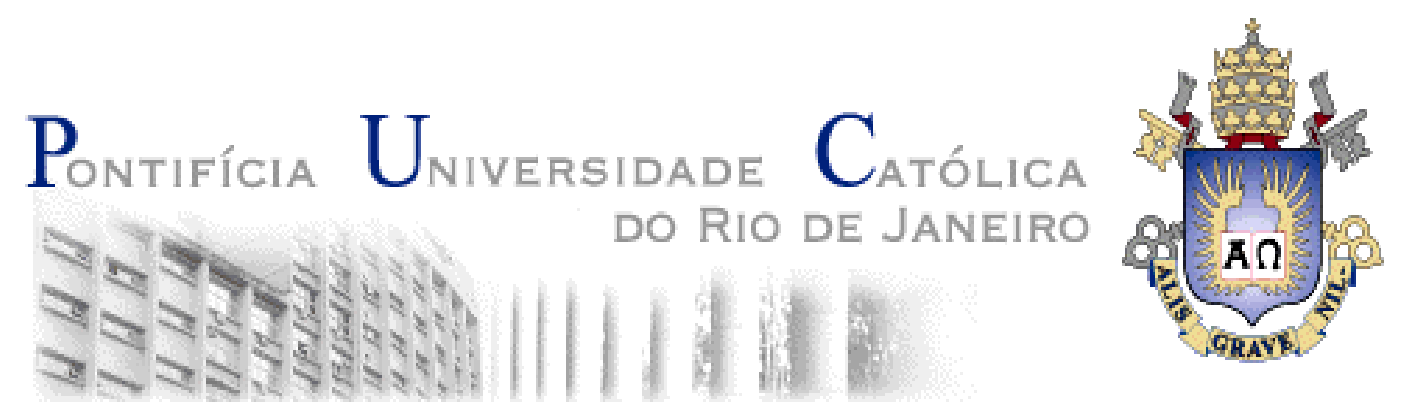

Danny Hernán Zambrano Carrera

Movimento de Rotação Sem Restrição de um Corpo Rígido

Tese apresentada como requisito parcial para obtenção do título de Doutor pelo Programa de PósGraduação em Engenharia Mecânica da PUC - Rio.

Orientador: Prof. Hans Ingo Weber 


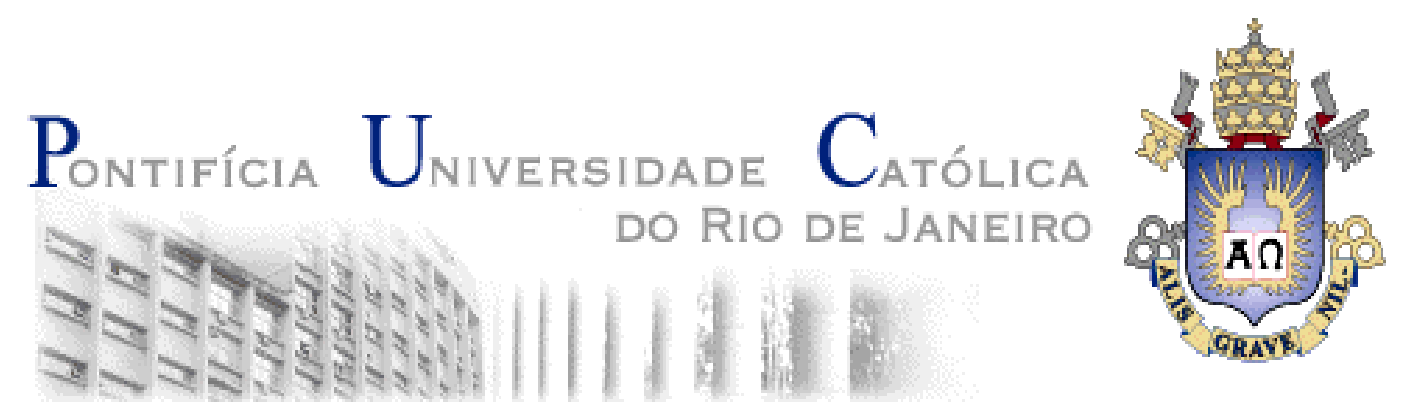

Danny Hernán Zambrano Carrera

\section{Movimento de Rotação Sem Restrição de um Corpo Rígido}

Tese apresentada como requisito parcial para obtenção do título de Doutor pelo Programa de Pós-Graduação em Engenharia Mecânica da PUC - Rio. Aprovada pela Comissão Examinadora abaixo assinada.

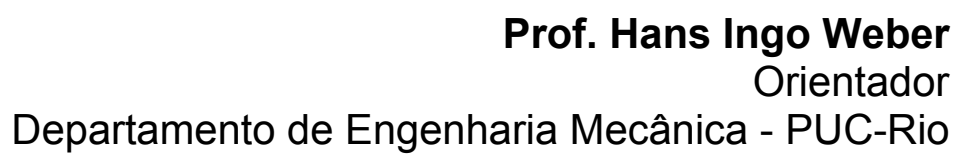

Prof. IImar Ferreira Santos

Technical University of Denmark, Department of Mechanical Engineering

Prof. José Manoel Balthazar

Departamento de Engenharia Mecânica - UNESP

Prof. Luciano Luporini Menegaldo Departamento de Engenharia Mecânica - IME

Prof. Marcelo Amorim Savi

Departamento de Engenharia Mecânica - UFRJ

Prof. Arthur Martins Barbosa Braga

Departamento de Engenharia Mecânica - PUC-Rio

Prof. José Eugenio Leal Coordenador Setorial do Centro Técnico Científico - PUC-Rio

Rio de Janeiro, 13 de Agosto de 2010 
Todos os direitos reservados. É proibida a reprodução total ou parcial do trabalho sem autorização da universidade, do autor e do orientador.

Danny Hernán Zambrano Carrera

Graduou-se em Engenharia Mecânica na Universidad Nacional de Ingeniería, UNI (Lima, Perú). Cursou mestrado na PUC - Rio em 2006, especializando-se em dinâmica veicular. Apresentou vários trabalhos em congressos nacionais e internacionais (COBEM, CILAMCE, PACAM, etc.) junto com o seu orientador durante os estudos de Doutorado.

Ficha Catalográfica

Carrera, Danny Hernán Zambrano

Movimento de rotação sem restrição de um corpo rígido / Danny Hernán Zambrano Carrera ; orientador: Hans Ingo Weber. - Rio de Janeiro, 2010.

120 f. : il. (color.) ; $30 \mathrm{~cm}$

Tese (Doutorado) - Pontifícia Universidade Católica do Rio de Janeiro, Departamento de Engenharia Mecânica, 2010.

Inclui bibliografia.

1. Engenharia mecânica - Teses. 2. Dinâmica não linear. 3. Ângulos de Cardan. 4. Quatérnios. 5. Orientação no espaço. 6. Limites de estabilidade. 7. Giroscópio. 8. Inércia. I. Weber, Hans Ingo. II. Pontifícia Universidade Católica do Rio de Janeiro. Departamento de Engenharia Mecânica. III. Título. 
Dedicado aos meus pais, Hernán e Sara, e a minha esposa, pelo apoio incondicional em toda minha caminhada. 


\section{Agradecimentos}

Gostaria de agradecer a todas as pessoas que tornaram possível a elaboração deste trabalho, em especial:

Aos meus pais, meus irmãos e toda a minha família por terem me incentivado a realizar este curso de Doutorado.

Ao professor Hans Ingo Weber pela orientação e constante incentivo.

Aos Professores membros da banca, pelos comentários e sugestões feitas.

Aos professores do Departamento de Engenharia Mecânica da PUC-Rio, pelos momentos compartilhados e conselhos a nível acadêmico.

Aos meus amigos e colegas do Departamento de Engenharia Mecânica da PUC-Rio, em especial para Monica Toledo, Wagner Epifânio e Ricardo Morrot, pelos momentos gratos.

À CAPES, à CNPq e à PUC-Rio, pelos auxílios concedidos, sem os quais este trabalho não poderia ter sido realizado. 


\section{Resumo}

Zambrano Carrera, Danny Hernán; Weber, Hans I. Movimento de Rotação Sem Restrição de um Corpo Rígido. Rio de Janeiro, 2010. 120p. Tese de Doutorado - Departamento de Engenharia Mecânica, Pontifícia Universidade Católica do Rio de Janeiro.

Um problema bem conhecido da Mecânica Clássica consiste no estudo do movimento de um corpo no espaço, especialmente quando o problema é conservativo e livre de forças. Este trabalho utiliza ferramentas modernas da Dinâmica para interpretar os movimentos com grande amplitude, ultrapassando os limites de estabilidade obtidos pelo conceito de Lyapunov. O problema da singularidade numérica que ocorre utilizando-se ângulos de Cardan pode ser eliminado com a descrição por quatérnios. A versatilidade dos quatérnios na Dinâmica é discutida, assim como a dificuldade do estudo do movimento próximo aos pontos de singularidade usando ângulos cardânicos. Enfatiza-se a influência dos momentos principais de inércia na estabilidade do movimento. Obtém-se um valor numérico da energia cinética mínima necessária para que o movimento atravesse o limite de estabilidade. O giroscópio Magnus é um instrumento educacional muito conveniente no estudo do movimento de um corpo livre no espaço. O rotor desse giroscópio representa um corpo em uma suspensão cardânica com anel externo e interno, o que dá ao corpo a liberdade de movimento necessária. Desenvolve-se nesta tese o modelo matemático de um corpo em suspensão cardânica, incluindo-se o atrito existente entre os componentes do sistema mecânico (além de considerar as inércias do rotor e dos anéis ou quadros). O problema da singularidade na descrição com rotações seqüenciais, que existe no caso de um corpo no espaço, é eliminado quando se considera a inércia dos quadros. Estuda-se o comportamento do giroscópio ao longo do tempo, sem outras restrições, considerando a perda de energia cinética devido ao atrito. Avalia-se também como a mudança dos momentos de inércia influencia a estabilidade do movimento do sistema.

\section{Palavras - chave}

Dinâmica não Linear; Ângulos de Cardan; Quatérnios; Orientação no Espaço; Limites de estabilidade; Giroscópio; Inércia. 


\section{Abstract}

Zambrano Carrera, Danny Hernán; Weber, Hans I. (Advisor). Libration and Tumbling of a Rigid Body. Rio de Janeiro, 2010. 120p. Doctorate Thesis - Departamento de Engenharia Mecânica, Pontifícia Universidade Católica do Rio de Janeiro.

A well known conservative problem in Classical Mechanics consists in the force free motion of a body in space. This work uses modern tools from Dynamics to interpret great amplitude movements crossing the limits of stability in the concept of Lyapunov. The numerical singularity that arises with the use of Cadan angles can be eliminated with quaternion representation. The versatility of quaternions in Dynamics is discussed, as well as the difficulty in investigating the motion near to singularity points when using cardanic angles. The influence of the principal moments of inertia on the stability of the motion is discussed. A numerical value for the minimal kinetic energy to cross the stability border is obtained. The Magnus Gyroscope is an educational instrument, very convenient in the study of the motion of a free body in outer space. The rotor of this gyroscope represents the body on a cardanic suspension with outer and inner ring, which gives the body the necessary freedom of motion. In this work a mathematical model of a body in cardanic suspension is generated, including friction between gimbals and rotor (besides considering the inertia of these components). The singularity problem in the free body solution is eliminated when the inertia of the gimbals is considered. Long term behavior of the unrestricted motion is investigated, considering the loss of kinetic energy due to friction. It is also shown how the change of moments of inertia due to the gimbals influences the stability of the motion of system.

\section{Keywords}

Nonlinear Dynamics; Cardan Angles; Quaternions; Orientation in Space; Limits of Stability; Gyroscope; Inertia. 


\section{Sumário}

1. Introdução 16

1.1. Histórico 16

1.2. Revisão bibliográfica 20

1.3. Terminologia 22

1.3.1. Velocidade angular 22

1.3.2. Matriz de transformação de coordenadas 23

1.3.3. Matriz de rotação 24

1.3.4. Quatérnios 26

1.3.5. Solução do problema inverso 27

1.3.6. Estabilidade 29

1.4. Objetivo 29

1.5. Descrição da tese 30

2. Descrição do sistema 31

2.1. Visualização do movimento 35

3. Modelo Matemático com ângulos seqüenciais 37

3.1. Corpo no espaço 39

3.1.1. Condição inicial do corpo no espaço 43

3.2. Sistema conservativo 46

3.2.1. Condição inicial do sistema conservativo 50

3.3. Sistema com atrito $\quad 51$

3.4. Trajetória do eixo do rotor 58

4. Modelo Matemático com Quatérnios 59

4.1. Corpo no espaço 59

4.1.1. Condição inicial do corpo no espaço 63

4.2. Trajetória do eixo do rotor 65 
5. Resultados Numéricos e Discussão das Simulações 66

5.1. Corpo no espaço 66

5.1.1. Bacia de atração para o corpo no espaço 70

5.1.2. Singularidade no bordo de estabilidade 79

5.1.3. Estudo de caos no corpo no espaço 83

5.2. Sistema conservativo de três corpos 89

5.2.1. Caos no sistema conservativo 96

5.3. Sistema com atrito 98

6. Vídeo e simulações 104

7. Conclusões 111

8. Referências Bibliográficas 116

Anexo: Triângulo de Magnus e Instabilidade 119 


\section{Lista de Figuras}

Figura 1.1: Rotação em torno de um eixo 25

Figura 2.1: Giroscópio de Magnus 31

Figura 2.2: Corpos que compõem o Giroscópio 32

Figura 2.3: Giroscópio no laboratorio 33

Figura 2.4: Corpo no espaço $\quad 34$

Figura 2.5: Giroscópio desenhado no programa OpenGL 35

Figura 3.1: Quantidade de movimento angular durante o impacto $\quad 37$

Figura 5.1: Gráfico da órbita $\alpha$ vs $\beta$, e trajetória espacial. Caso $1 \quad 67$

Figura 5.2: Ângulos cardânicos e as suas velocidades. Caso 1

Figura 5.3: Componentes do Quatérnio e suas derivadas. Caso $1 \quad 68$

Figura 5.4: Velocidade angular do corpo, escrito no $S R(F)$. Caso $1 \quad 68$

Figura 5.5: Gráfico da órbita $\alpha$ vs $\beta$, e trajetória espacial. Caso $2 \quad 69$

Figura 5.6: Ângulos cardânicos e as suas velocidades. Caso $2 \quad 69$

Figura 5.7: Componentes do quatérnio e suas derivadas. Caso $2 \quad 69$

Figura 5.8: Velocidade Angular do corpo, escrito no SR(F). Caso $2 \quad 70$

Figura 5.9: Bacia de atração segundo os ângulos cardânicos 71

Figura 5.10: Movimento cruzando o borde de estabilidade 72

Figura 5.11: Bacia de atração em quatérnios 73

Figura 5.12: Bacia de atração em quatérnios no plano $\varphi-\theta \quad 74$

Figura 5.13: Energia normalizada em função de $\alpha$ e $\gamma$, achatado $\quad 75$

Figura 5.14: Energia cinética e normalizada, corpo achatado 76

Figura 5.15: Energia cinética e normalizada, corpo alongado $\quad 77$

Figura 5.16: Triângulo de Magnus para um corpo no espaço 78

Figura 5.17: Órbita $\alpha$ vs $\beta$, bacia de atração em quatérnios. Caso 379

Figura 5.18: Ângulos cardânicos e quatérnios. Caso $3 \quad 80$

Figura 5.19: Trajetória espacial, comparação. Caso $3 \quad 80$

Figura 5.20: Órbita $\alpha$ vs $\beta$, bacia de atração em quaternios. Caso $4 \quad 81$

Figura 5.21: Ângulos cardânicos e quatérnios. Caso 4

Figura 5.22: Trajetória espacial, comparação. Caso 4

Figura 5.23: Velocidade Angular do corpo, escrito no SR(F). Caso $4 \quad 82$ 
Figura 5.24: Função de $\gamma_{(\alpha, \beta)}^{\prime}$ para $\gamma=0$

Figura 5.25: Função de $E E_{(\alpha, \beta)}$ para $\gamma=\pi / 2$

Figura 5.26: Plano-fase dos ângulos cardânicos 84

Figura 5.27: Mapa de Poincaré dos ângulos reduzidos: $-\pi / 2$ até $\pi / 2 \quad 85$

Figura 5.28: Diagramas de bifurcação dos ângulos 85

Figura 5.29: Expoente de Lyapunov da coordenada $z$ e ângulo $\alpha \quad 86$

Figura 5.30: Plano-fase das componentes dos quaternio: $v_{x}$ e $l \quad 86$

Figura 5.31: Mapa de Poincaré do quatérnio, para $z=0$

Figura 5.32: Mapa de Poincaré do quaternio, para $\mathrm{v}_{\mathrm{z}} . l=0$

Figura 5.33: Diagramas de bifurcação dos quatérnios 88

Figura 5.34: Expoente de Lyapunov da coordenada $z$ e $v_{x}$

Figura 5.35: Órbita $\alpha-\beta$, e trajetória espacial. Caso 5

Figura 5.36: Ângulos cardânicos e as suas velocidades. Caso 590

Figura 5.37: Energia e Quantidade de movimento angular. Caso 590

Figura 5.38: Bacia de atração para o sistema conservativo 91

Figura 5.39: Energia cinética e normalizada, para tipos de corpos 91

Figura 5.40: Triângulo de Magnus do Sistema conservativo $(y)$

Figura 5.41: Triângulo de Magnus do Sistema conservativo $(x) \quad 93$

Figura 5.42: Diagrama de Bifurcação para o quadro externo 93

Figura 5.43: Diagrama de Bifurcação para o quadro interno 93

Figura 5.44: Áreas de instabilidade em inércia dos quadros 95

Figura 5.45: Energia do sistema para inércia dos quadros $(y$ e $x$ ) 95

Figura 5.46: Diagrama plano-fase dos ângulos cardânicos 96

Figura 5.47: Diagrama de Poincaré no $\alpha-\beta$, para velocidades nulas $\quad 97$

Figura 5.48: Expoente de Lyapunov do Sistema conservativo 97

Figura 5.49: Diagramas de Bifurcação para k 98

Figura 5.50: Diagramas de Bifurcação para $\mathrm{k} \quad 98$

Figura 5.51: Orbita $\alpha-\beta$ eliminando o atrito nas juntas 99

Figura 5.52: Órbita $\alpha-\beta$, e trajetória espacial. Caso $6 \quad 100$

Figura 5.53: Ângulos cardânicos e as suas velocidades. Caso $6 \quad 101$

Figura 5.54: Energia e Quantidade de movimento angular. Caso $6 \quad 101$

Figura 5.55: Diagrama Plano-fase do ângulo $\alpha$. Caso $6 \quad 102$

Figura 5.56: Bacias de atração do sistema com atrito 102

Figura 6.1: Orbita do rotor achatado $\left(\Delta H_{x} / H_{G}=0.15\right)$ 
Figura 6.2: Orbita do rotor achatado $\left(\Delta H_{x} / H_{G}=0.7\right) \quad 106$

Figura 6.3: Orbita do rotor alongado $\left(\Delta H_{x} / H_{G}=0.3\right) \quad 106$

Figura 6.4: Precessão retrograda no giroscópio com rotor achatado 107

Figura 6.5: Precessão direta no giroscópio com rotor alongado 108

Figura 6.6: Órbitas do rotor não axissimétrico achatado 109

Figura 6.7: Mudanças de hemisfério, sistema com atrito 110

Figura A: Principais características do triângulo de Magnus 119

Figura B: Região instável ou estável segundo a configuração inercial 120 


\section{Lista de Tabelas}

Tabela 2.1. Propriedades físicas dos corpos, Giroscópio

Tabela 6.1. Momentos de inércia do giroscópio do laboratório 


\section{Lista de Símbolos}

\section{Símbolos Romanos}

RS Sistema referencial

$\tilde{\mathbf{r}} \quad$ Matriz til associada ao vetor $\mathbf{r}$

F Sistema referencial fixo no espaço

$Q \quad R \quad S \quad$ Sistemas referenciais móveis

${ }^{F} \mathbf{T}^{S} \quad$ Matriz transformação de coordenadas entre os SR: (S) e (F)

$e_{s} \quad$ Eixo de simetria do rotor, ou eixo de simetria

$e_{r} \quad$ Eixo de rotação, paralelo ao vetor velocidade angular

p Vetor de rotação

v $l$ Vetor e escalar, componentes do quatérnio

$v_{x} \quad v_{y} \quad v_{z} \quad$ Componentes do vetor $\mathbf{v}$

q Quatérnio, vetor $4 \times 1$

$\mathbf{q}^{*} \quad$ Conjugado do quatérnio $\mathbf{q}$

q $\quad$ Matriz $4 \times 4$ associada ao quatérnio $\mathbf{q}$

$\mathbf{H}_{G} \quad$ Quantidade de movimento angular antes do impacto

$\mathbf{h}_{G_{0}} \quad$ Quantidade de movimento angular depois do impacto

$\Delta H y \quad$ Variação da quantidade de movimento angular em $y_{0}$

$\Delta H x \quad$ Variação da quantidade de movimento angular em $x_{0}$

$\begin{array}{lllll}x & y & z & \text { Coordenadas do sistema referencial fixo }\end{array}$

M Momento ou torque

$t \quad$ Tempo

I Matriz inércia

Ec Energia cinética

EE Energia cinética normalizada

$\mu_{1} \quad \mu_{2} \quad$ Momentos de inércia do rotor, normalizados

$\mu_{x} \quad \mu_{p} \quad$ Momentos de inércia dos quadros cardânicos, normalizados

$A \quad B \quad C \quad D$ Parâmetros adimensionais 


\section{Símbolos Gregos}

$\theta \quad$ Ângulo de giro em torno do vetor de rotação

$\varphi \quad$ Ângulo do cone, entre o vetor de rotação e eixo de simetria

$\phi \quad$ Ângulo usado na definição do Quatérnio

$\alpha \quad \beta \quad \gamma \quad$ Angulos de Cardan

$\dot{\alpha} \quad \dot{\beta} \quad \dot{\gamma} \quad$ Derivadas temporais dos ângulos de Cardan

$\alpha^{\prime} \quad \beta^{\prime} \quad \gamma^{\prime} \quad$ Derivadas adimensionais dos ângulos de Cardan

$\alpha^{\prime \prime} \quad \beta^{\prime \prime} \quad \gamma^{\prime \prime} \quad$ Segunda derivada adimensional dos ângulos de Cardan

$\Omega \quad$ Velocidade angular do corpo antes do impacto

$v \quad$ Velocidade angular equivalente do corpo depois do impacto

$\tau \quad$ Parâmetro adimensional

${ }^{F} \boldsymbol{\omega}_{S} \quad$ Velocidade angular do corpo, escrito no sistema referencial (F)

$\kappa_{F Q} \quad \kappa_{Q R} \quad \kappa_{R S}$ Coeficientes normalizados de atrito viscoso 